\title{
Optical assessment of pathology in surgically resected tissues
}

\author{
Ashley Laughney*a, Venkataramanan Krishnaswamy ${ }^{\mathrm{a}}$, Wendy A. Wells ${ }^{\mathrm{b}}$, Olga M. Conde ${ }^{\mathrm{c}}$, Keith D. \\ Paulsen ${ }^{\mathrm{a}}$, Brian W. Pogue ${ }^{\mathrm{a}}$, \\ ${ }^{a}$ Thayer School of Engineering at Dartmouth College, 8000 Cummings Hall, Hanover, NH 03755 \\ ${ }^{b}$ Department of Pathology, Dartmouth-Hitchcock Medical Center, \\ 1 Medical Center Drive, Lebanon, NH 03756 \\ ${ }^{c}$ University of Cantabria, Avda. De los Castros S/N, Santander 39005, Spain \\ *ashley.laughney@dartmouth.edu
}

\begin{abstract}
Multi-spectral spatially modulated light is used to guide localized spectroscopy of surgically resected tissues for cancer involvement. Modulated imaging rapidly quantifies near-infrared optical parameters with sub-millimeter resolution over the entire field for identification of residual disease in resected tissues. Suspicious lesions are further evaluated using a spectroscopy platform designed to image thick tissue samples at a spatial resolution sensitive to the diagnostic gold standard, pathology. MI employs a spatial frequency domain sampling and model-based analysis of the spatial modulation transfer function to interpret a tissue's absorption and scattering parameters at depth. The spectroscopy platform employs a scanning-beam, telecentric dark-field illumination and confocal detection to image fields up to $1 \mathrm{~cm}^{2}$ with a broadband source $(480: 750 \mathrm{~nm})$. The sampling spot size $(100 \mu \mathrm{m}$ lateral resolution) confines the volume of tissue probed to within a few transport pathlengths so that multiple-scattering effects are minimized and simple empirical models may be used to analyze spectra. Localized spectroscopy of Intralipid and hemoglobin phantoms demonstrate insensitivity of recovered scattering parameters to changes in absorption, but a non-linear dependence of scattering power on Intralipid concentration is observed due to the phase sensitivity of the measurement system. Both systems were validated independently in phantom and murine studies. Ongoing work focuses on assessing the combined utility of these systems to identify cancer involvement in vitro, particularly in the margins of resected breast tumors.
\end{abstract}

Keywords: scatter, broadband spectroscopy, modulated imaging, breast cancer, surgery, pathology

\section{INTRODUCTION}

Breast conserving therapy (BCT), which includes local excision and radiation treatment to the breast, has been the standard of care for early breast cancers (Stage 0-II), since five major prospective, randomized trials in North American and Europe demonstrated that the long-term survival after BCT is equivalent to that of radical mastectomy for most patients when surgical margins are clear of residual disease $\mathrm{e}^{1-5}$. Most of the 250,000 women annually diagnosed with breast cancer are eligible for BCT, resulting in approximately 165,000-180,000 conservative surgeries each year . Following breast conservation, the strongest risk factor for local recurrence and mortality is a positive resection margin (tumor cells on ink). Therefore, if a margin is positive or close (cancer present within 1-2mm of margin surface), the patient is advised to undergo re-excision surgery to achieve clear margins ${ }^{7,8}$. Margin status is currently evaluated postoperatively by microscopic evaluation of pathology in small, representative pieces of tissue. While sampled tissues are adequate for assessing tumor type, grade and receptor status, they are insufficient for evaluating important prognostic factors like disease extent, multi-focality and heterogeneity.

The fundamental challenge encountered when applying spectral techniques to margin assessment is the need for microscopic resolution over a wide field. Techniques that probe large volumes of tissue rely on the assumption that ultra-structural malignant transformations provide disease-specific contrast in volume-averaged measures, but breast tissue is markedly heterogeneous, which makes distinguishing small foci of cancer within the spectrum of normal tissue challenging ${ }^{9-11}$. Most spectral tools developed for margin assessment involve fiber bundles that cannot sample the full tissue field at a resolution sufficient to account for specimen heterogeneity ${ }^{12,13}$. So while spectroscopy methods like

Biomedical Applications of Light Scattering V, edited by Adam P. Wax, Vadim Backman,

Proc. of SPIE Vol. 7907, 79070P · @ 2011 SPIE - CCC code: 1605-7422/11/\$18 - doi: 10.1117/12.874059 
fluorescence, diffuse reflectance and Raman spectroscopy are responsive to the tissue's biochemical and ultra-structural state $^{14-18}$; these techniques minimally sample tissue pathology.

A broadband spectroscopy platform was developed to image thick tissue samples at a resolution sensitive to the diagnostic gold standard, pathology ${ }^{19}$. Tissue samples were raster-scanned across a static beam using a motorized stage and the sampling spot size $(100 \mu \mathrm{m}$ lateral resolution) confined the volume of tissue probed to within a few transport pathlengths so that multiple-scattering effects were minimized and simple empirical models parameterized the spectra. A k-Nearest Neighbor (k-NN) classifier was trained using parameters extracted from the localized scattering spectrum, automating diagnosis of benign and malignant breast pathologies in situ with a sensitivity and specificity of $91 \%$ and $77 \%$ respectively. Performance of the classifier was validated in 67,000 spectra from 29 excised breast tissues ${ }^{20}$. This initial study characterized the spectral response of breast pathologies, but the system's utility was limited by its long data acquisition times and restricted sampling field.

Data acquisition times have been improved via development of a dark-field in situ scanning-beam spectroscopy platform that rapidly sample fields up to $1 \mathrm{~cm}^{2}$. Nonetheless, candidates for BCT may have tumors up to $5 \mathrm{~cm}$ in diameter and spectral analysis of the full tumor field is not feasible. To overcome the fundamental tradeoff between microscopic resolution and field side, a multi-modal approach was employed. Modulated imaging (MI), a non-contact and nonscanning technique that rapidly samples wide-field optical properties at sub-millimeter resolution, was used to guide dense spectral sampling using the scanning-beam spectroscopy platform ${ }^{21}$. Scanned ROI are then correlated with pathology to validate the combine utility of these tools to assess tumor margin status. Figure (1) presents a schematic of this methodology.

\section{METHODOLOGY}

(a)

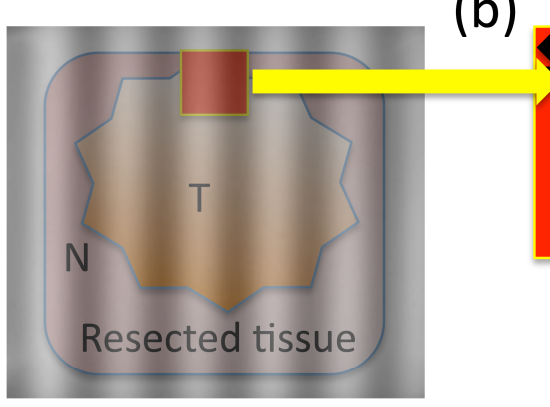

(b)

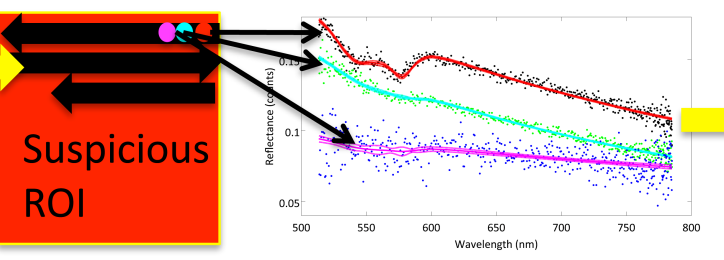

(c)

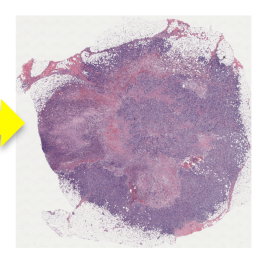

Figure 1 Schematic of the optical assessment methodology: (a) sample full-field optical parameters with modulated imaging, (b) broadband spectroscopy in suspicious ROI, (c) classify spectral parameters according to pathology diagnosis.

\subsection{Multi-spectral spatially modulated light to guide spectroscopy}

Modulated imaging (MI) employs spatial frequency domain sampling and model-based analysis of the spatial modulation transfer function (s-MTF) to interpret a tissue's optical properties at depth. A compact modulated imaging system was purchased from Modulated Imaging Inc., to map a tissue's absorption and scattering parameters at depth using spatially modulated plane wave projections in the NIR $(658,730,850,970 \mathrm{~nm})$. System specifications and theory are detailed in the references ${ }^{22,23}$.

Sinusoidal patterns of varying spatial frequency are projected onto the sample using a projection system and digital micro-mirror device. A one-dimensional modulation is generated in the $\mathrm{x}$-direction to simplify the homogenous model of diffuse reflectance and a 16-bit CCD camera captures the diffusely reflected photons. Specular reflections are minimized by illuminating at a small angle to normal incidence and by using crossed linear polarizers. Each spatial frequency is projected at three phase angles for amplitude demodulation of the $\mathrm{AC}$ and $\mathrm{DC}$ signals ${ }^{24}$. A Siloxane $\mathrm{TiO}_{2}$ reflectance $^{2}$ standard is used to calibrate for intensity fluctuations and spatial non-uniformity in the illumination and imaging systems. Each spatial frequency projection is repeated at four NIR wavelengths using high power LED sources (658, 730,850 and $970 \mathrm{~nm}$ ) and interference filters are employed to constrain the detection waveband. Diffuse reflectance is 
mapped as a function of frequency using the spatial Fourier transform of modulated images obtained at varying spatial frequencies. The steady state diffusion equation reduces to a second-order Helmholtz equation for fluence as a function of depth when assuming a sinusoidal source incident upon a homogenous, linear media ${ }^{22}$. Multi-spectral measures allow the use of a power law modified by Beer's law to fit for the reduced scattering coefficient and chromophore concentrations. The near-infrared wavelengths enable quantification of the concentrations of oxygenated and deoxygenated hemoglobin, water, adipose and cellular scattering. Optical properties are recovered using a rapid twofrequency look up table (LUT), constructed using cubic spline interpolation of the forward-model data at two frequencies.

\subsection{Scanning-beam broadband spectroscopy in suspicious ROI}

Suspicious lesions identified by MI parameter maps are imaged at a higher resolution using the scanning-beam spectroscopy platform. Its illumination and detection spot size is laterally constrained to $100 \mu \mathrm{m}$, minimizing multiplescattering effects so that simple empirical models may be used to parameterize the spectra. Full spectra are acquired at each $100 \mu \mathrm{m}$-diameter pixel for thick tissue samples in a $1 \mathrm{~cm}^{2}$ field. The spectroscopy platform is detailed in the reference $^{21}$. It combines a broadband telecentric scanning design with dark-field illumination/detection for efficient rejection of specular light, while maintaining a consistent sampling geometry across the image field. A tungsten halogen lamp is used as the source $(480: 750 \mathrm{~nm})$ with an exposure time of $300 \mathrm{~ms}$ for pancreas tissues. Tissue acquisition took approximately 40min, during which time the tissue was hydrated with phosphate buffer saline. Much faster exposure times are possible with a broadband super continuum laser source, which is currently being integrated into the system. All measures are background subtracted and referenced to gray Spectralon (Labsphere Inc) on a point-by-point basis. An empirical approximation to Mie theory modified by a Beer-Lambert attenuation factor to account for local absorption by chromophores was sufficient to parameterize spectra ${ }^{20}$.

$$
\left.R_{\lambda}=A\left[f \lambda^{-b}+(1-f) \lambda^{-4}\right)\right] \exp ^{-\Gamma_{\lambda}[H b T]\left(f S O_{2} \varepsilon_{H b O_{2}, \lambda}+\left(1-f S O_{2}\right) \varepsilon_{H b, \lambda}\right)}
$$

Here, the wavelength-dependence of the reduced scattering coefficient is described by a combination of Rayleigh and Mie theory. $A, b$ and $f$ are the scattering amplitude, scattering power, and Mie contribution to scatter respectively. The scattered reflectance is exponentially attenuated due to chromophore absorption. The absorption coefficient is defined as the product of each chromophore concentration with its wavelength-dependent molar extinction coefficient. The primary tissue absorbers in the 480-750nm waveband are oxygenated and deoxygenated hemoglobin. Their concentrations are coupled by the parameters: total hemoglobin, $[\mathrm{HbT}]$ and the oxygen saturation factor, $\mathrm{fSO}_{2}$. The molar extinction spectra of these chromophores are given by $\varepsilon_{\mathrm{HbO}_{2}}(\lambda)$ and $\varepsilon_{\mathrm{Hb}}(\lambda)$ respectively ${ }^{25} . \Gamma_{\lambda}$ represents the mean optical pathlengths, which depends on both the measurement geometry and the absorption and scattering properties of the tissue. Scanned ROI are then correlated with the diagnostic gold standard, pathology, to assess the ability of these tools to assess the tissue's disease state.

\section{DATA}

\subsection{Phantom Data}

A series of Intralipid and hemoglobin phantoms were imaged to characterize the spectroscopy system's response to changes in absorption and scattering. A series of scattering phantoms with [ $\left[\begin{array}{llllll}5 & 4 & 3 & 2 & 1 & 0.5\end{array}\right] \%$ Intralipid were serially diluted with porcine blood, with a maximum concentration of $30 \mathrm{uM}$ hemoglobin. All measures were background subtracted and referenced to $5 \%$ Intralipid on a point-by-point basis. The exposure time was $100 \mathrm{~ms}$. Figure 2 (a) shows the nonlinear relationship between the calculated ${ }^{26}$ and recovered reduced scattering coefficient for varying concentrations of hemoglobin. Recovered scattering parameters are independent to changes in absorption, but a nonlinear dependence on scatterer concentration is observed due to the phase sensitivity of the measurement system. Figure 2(b) shows the recovered product of total hemoglobin concentration and transport path length, as a function of true hemoglobin concentration, at the five concentrations of Intralipid. The box and whisker plots illustrate the spread in recovered absorption parameter for all concentrations of Intralipid. 

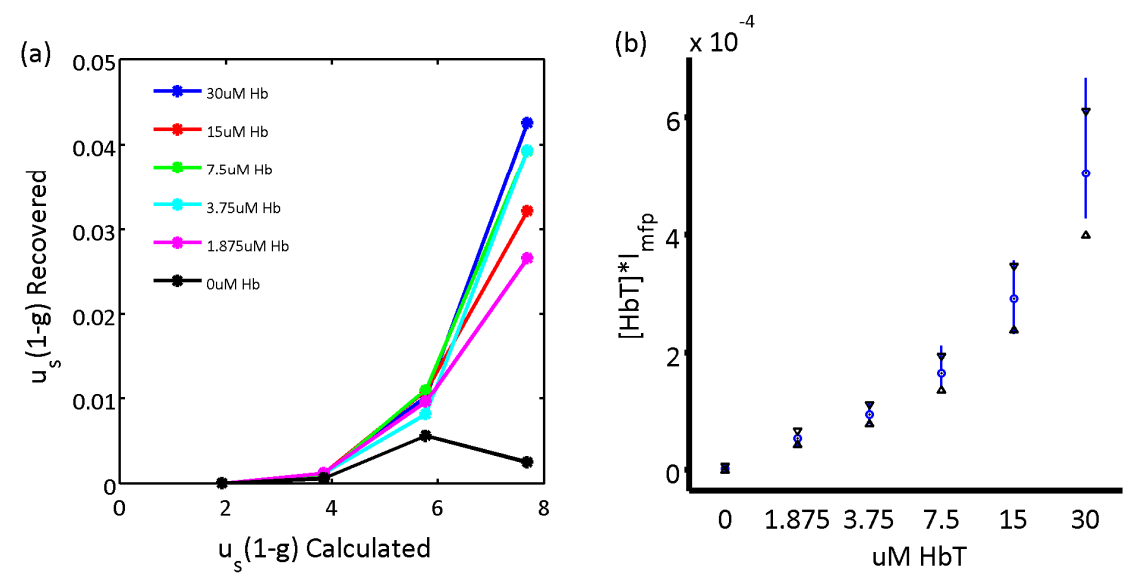

Figure 2 (a) Calculated vs. recovered reduced scattering coefficient as a function of variation in total hemoglobin and Intralipid concentration; (b) recovered path length times total hemoglobin as a function of total hemoglobin concentration.

Box and whisker plots indicate variation in recovered values from Intralipid concentrations varying from 0.5 to $5 \%$.

Similarly, phantom studies are being used to characterize the spectral response of the modulated imaging system.

\subsection{Tissue Data}

To assess the combined use of modulated imaging and the scanning beam spectroscopy platform to characterize the pathology of excised tissues, four normal pancreas and four pancreas tumors mouse were imaged in a murine model in vitro. The tissue was hydrated with a phosphate buffer solution and imaged immediately after excision. Figure 3(a-c) shows the optical maps of a murine pancreas on a diffusive background generated by modulated imaging. A suspicious region of interest is highlighted in blue. Figure 3(d) shows the parameter maps generated by the scanning-beam spectroscopy platform within the suspicious field of view and Figure 3(e) shows its corresponding pathology.

(a)

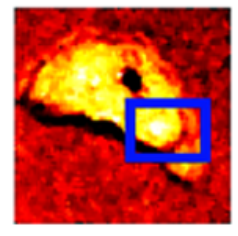

(d) (b)
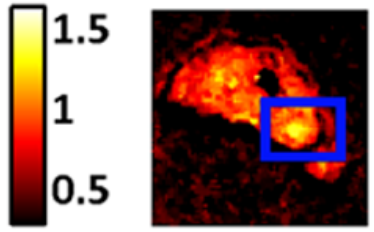

(c)

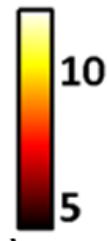

(e)

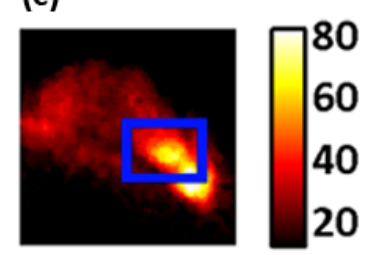

20

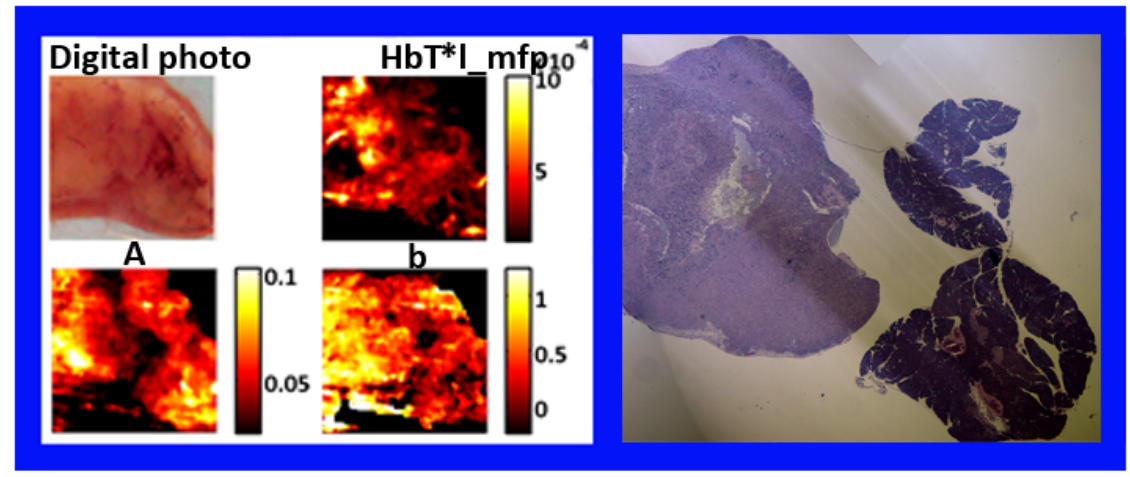

Figure 3 (a) Scattering power, (b) log of the scattering amplitude and (c) total hemoglobin recovered by the modulated imaging system. (d) Optical maps generated by the scanning beam spectroscopy platform with a digital photograph of the tissue ROI in its upper left corner; (e) corresponding histology. 
Optical maps were correlated to pathology and the spectral response of normal, tumor and necrotic pathologies were assessed. Correlation with microscopic features was not possible because the histopatholopic processing of the murine tissues resulted in severe artifacts that limited co-registration between pathology and recovered optical maps. In the future, inked pins will be used to mark imaged fields of view to improve co-registration.

\section{RESULTS}

Figure 4(a) shows the spectral response of normal, tumor and necrotic tissue in the murine pancreas. Greater scattering slope is observed in the tumor and necrotic pathologies as compared to normal pathologies. Figure 4(b-d) illustrates clustering of the spectral remission parameters for all pixels according to pathologic diagnosis.

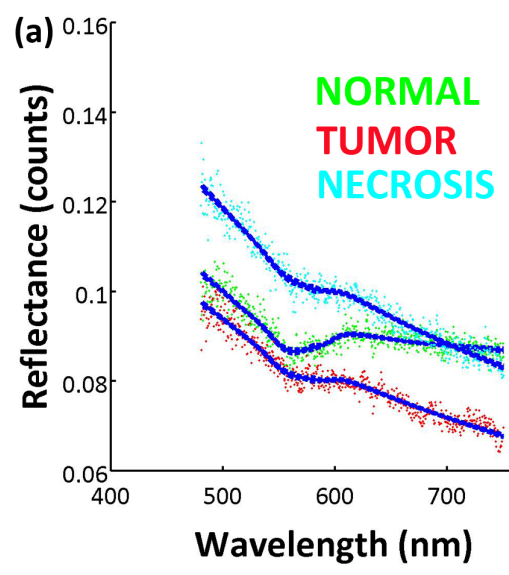

(c)

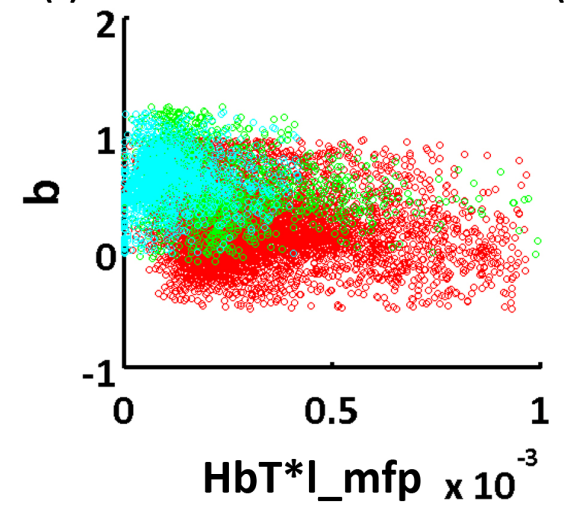

(b)

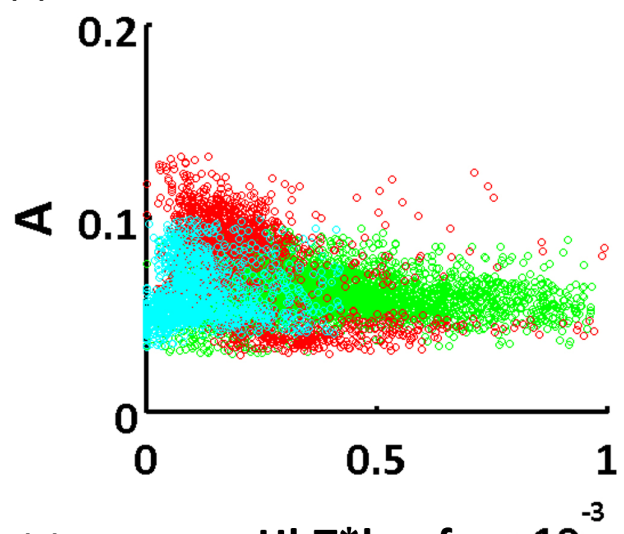

(d)

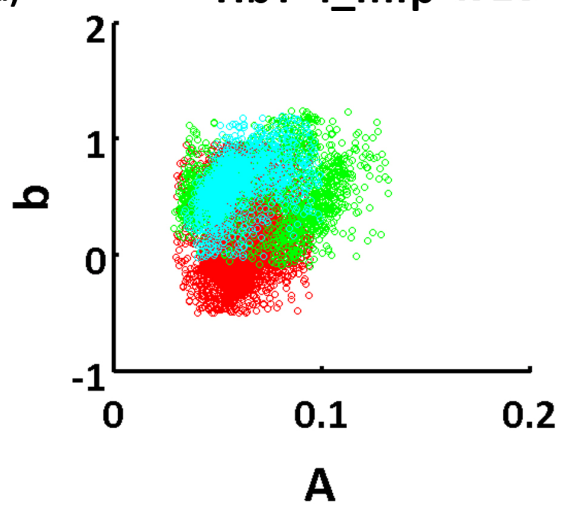

Figure 4 (a) Spectral response of normal, tumor and necrotic pathologies. (b-d) Two-dimensional scattering plots of the recovered optical parameters. Average scattering irradiance is not displayed because it demonstrates the same trend as the scattering amplitude.

This type of dataset may be used to train a classification algorithm to identify pathologies based spectral features, as demonstrated previously using breast tissue ${ }^{20}$. Only the scattering power distribution was normal as determined by looking at the normal data probability plot for all parameters and diagnostic categories. Normality is expected to improve with increasing sample size. A Kruskal-Wallis test was used to compare the medians for each parameter distribution according to diagnostic category. Figure 5(a-d) illustrates box plots of each parameter distribution according to diagnosis. 

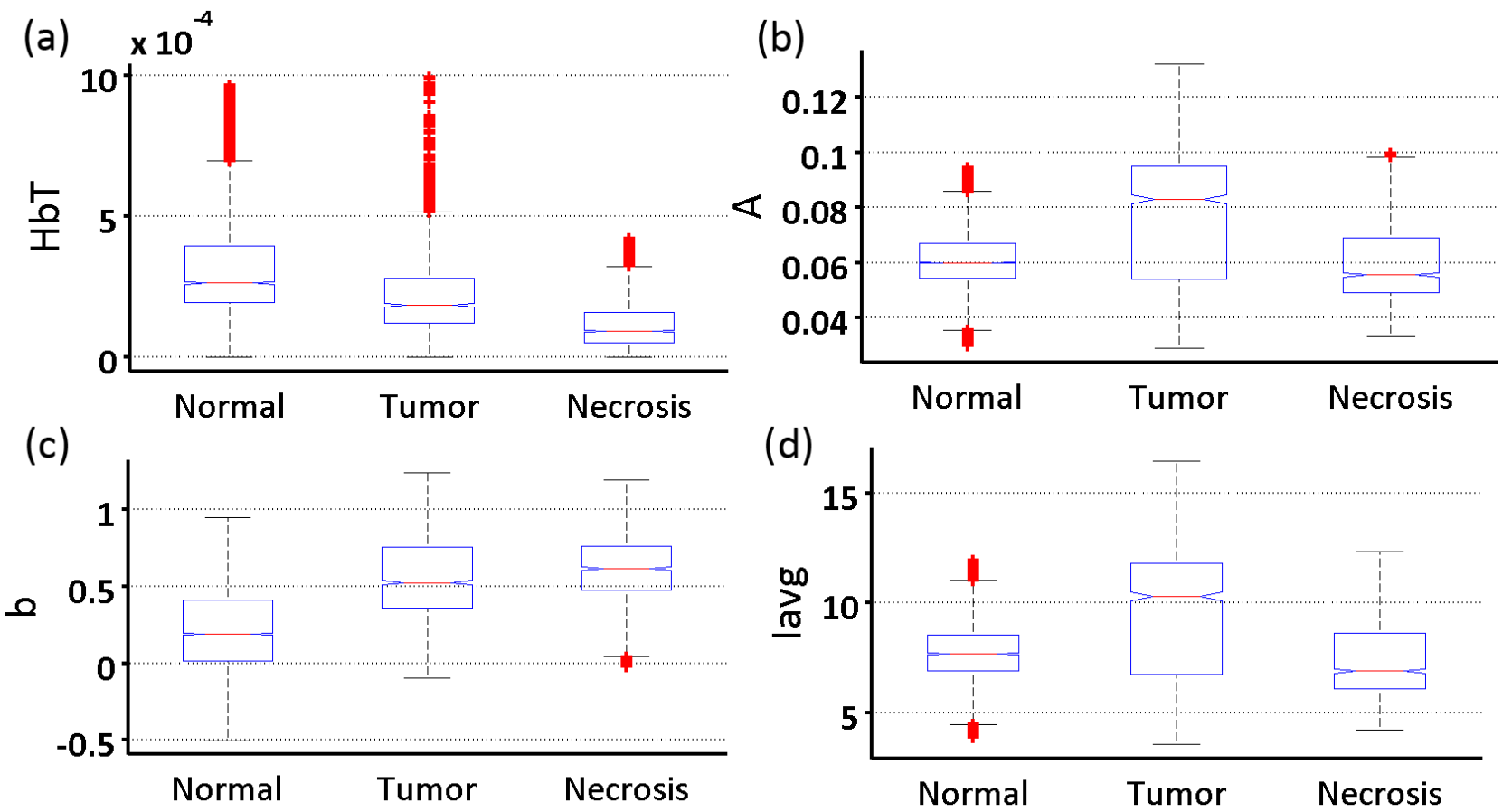

Figure 5 (a) distribution of recovered hemoglobin values, (b) scattering amplitude, (c) scattering power and (d) average scattering irradiance for all diagnostic categories. p-values for all approached 0 , indicating a statistically significant difference between the medians of the distributions.

The computed p-values were all much less than 0.05 , indicating that the sample medians are significantly different.

\section{CONCLUSIONS}

Breast tissue is markedly heterogeneous, therefore wide field of view imaging is necessary to assess disease extent. Additionally, localized spectroscopy is needed to identify small foci of cancer within a heterogeneous background and to correlate measures with the diagnostic gold standard, pathology. The proposed methodology uses NIR spatially modulated light to guide localized spectroscopy of excised tissues. The modulated imaging system samples the full tissue field, rapidly, in a non-contact and non-scanning way. The scanning-beam spectroscopy platform rapidly rasterscans thick tissue samples in $1 \mathrm{~cm}^{2}$ suspicious fields of view, recovering broadband spectra from each 100-micron diameter pixel. The systems have been validated independently and this multi-modal methodology was assessed in murine studies. Future work involves assessment of the combined utility of the systems identify residual cancer in resected breast tissues.

\section{ACKNOWLEDGEMENTS}

This work has been funded by NIH grants PO1 CA80139, 5R21RR024411-02 and the DoD CDMRP grant BC093811.

\section{REFERENCES}

[1] U. Veronesi, N. Cascinelli, L. Mariani, M. Greco, R. Saccozzi, A. Luini, M. Aguilar and E. Marubini, "Twenty-year follow-up of a randomized study comparing breast-conserving surgery with radical mastectomy for early breast cancer," New Engl J Med 347 (16), 1227-1232 (2002).

[2] R. Arriagada, M. G. Le, F. Rochard and G. Contesso, "Conservative treatment versus mastectomy in early breast cancer: Patterns of failure with 15 years of follow-up data," J Clin Oncol 14 (5), 1558-1564 (1996).

[3] J. A. Jacobson, et al., "Ten-Year Results of a Comparison of Conservation with Mastectomy in the Treatment of Stage I and II Breast Cancer," New Engl J Med 332 (14), 907-911 (1995). 
[4] J. A. van Dongen, et al., "Long-term results of a randomized trial comparing breast-conserving therapy with mastectomy: European Organization for Research and Treatment of Cancer 10801 trial," J Natl Cancer I 92 (14), 1143-1150 (2000).

[5] M. Blichert-Toft, C. Rose, J. A. Andersen, M. Overgaard, C. K. Axelsson, K. W. Andersen and H. T. Mouridsen, "Danish randomized trial comparing breast conservation therapy with mastectomy: six years of life-table analysis. Danish Breast Cancer Cooperative Group," J Natl Cancer Inst Monogr (11), 19-25 (1992).

[6] J. Q. Brown, et al., "Optical Assesssment of Tumor Resection Margins in the Breast," IEEE JOURNAL OF SELECTED TOPICS IN QUANTUM ELECTRONICS 16 (3), 530-544 (2010).

[7] S. A. Khan and F. Eladoumikdachi, "Optimal Surgical Treatment of Breast Cancer: Implications for Local Control and Survival," Journal of Surgical Oncology 101 (8), 677-686 (2010).

[8] M. Kaufmann, M. Morrow, G. von Minckwitz, J. R. Harris and M. Biedenkopf Expert Panel, "Locoregional Treatment of Primary Breast Cancer Consensus Recommendations From an International Expert Panel," Cancer 116 (5), 1184-1191 (2010).

[9] S. Kennedy, et al., "Optical breast cancer margin assessment: an observational study of the effects of tissue heterogeneity on optical contrast," BREAST CANCER RESEARCH 12 (6), R91 (2010).

[10] Bydlon, et al., "Performance metrics of an optical spectral imaging system for intra-operative assessment of breast tumor margins," Opt Express 18, 8058 - 8076 (2010).

[11] T. M. Bydlon, et al., "Rapid Optical Imaging of Breast Tumor Margins: Final Results from a 100-Patient Clinical Study," Cancer Res 69 (24), 770s-771s (2009).

[12]A. Myakov, L. Nieman, L. Wicky, U. Utzinger, R. Richards-Kortum and K. Sokolov, "Fiber optic probe for polarized reflectance spectroscopy in vivo: Design and performance," JOURNAL OF BIOMEDICAL OPTICS 7 (3), 388-397 (2002).

[13] J. R. Mourant, T. J. Bocklage, T. M. Powers, H. M. Greene, M. H. Dorin, A. G. Waxman, M. M. Zsemlye and H. O. Smith, "Detection of Cervical Intraepithelial Neoplasias and Cancers in Cervical Tissue by In Vivo Light Scattering," Journal of Lower Genital Tract Disease 13 (4), 216-223 (2009).

[14] A. C. Lee, C. D. O. Pickard, M. R. S. Keshtgar, G. M. Briggs, M. Falzon, S. Lakhani, I. Bigio and S. G. Bown, "Elastic scattering spectroscopy for the diagnosis of breast cancer," Brit J Surg 89, 74-74 (2002).

[15]I. Bigio, et al., "Diagnosis of breast cancer using elastic-scattering spectroscopy: preliminary clinical results," J Biomed Opt 5, 221 - 228 (2000).

[16] A. Mahadevan-Jansen, M. F. Mitchell, N. Ramanujam, A. Malpica, S. Thomsen, U. Utzinger and R. RichardsKortum, "Near-infrared Raman spectroscopy for in vitro detection of cervical precancers," Photochem Photobiol 68 (1), 123-132 (1998).

[17]C. J. Frank, R. L. McCreery and D. C. B. Redd, "RAMAN-SPECTROSCOPY OF NORMAL AND DISEASED HUMAN BREAST TISSUES," Anal Chem 67 (5), 777-783 (1995).

[18] I. J. Bigio and J. R. Mourant, "Ultraviolet and visible spectroscopies for tissue diagnostics: Fluorescence spectroscopy and elastic-scattering spectroscopy," PHYSICS IN MEDICINE AND BIOLOGY 42 (5), 803-814 (1997).

[19] V. Krishnaswamy, Wells, W.A., Laughney, A.M., Samkoe, K.S.,Pogue, B.W., presented at the SPIE Biomedical Applications of Light Scattering III, San Jose, CA, USA, 2009 (unpublished).

[20] A. M. Laughney, V. Krishnaswamy, P. B. Garcia-Allende, O. M. Conde, W. A. Wells, K. D. Paulsen and B. W. Pogue, "Automated classification of breast pathology using local measures of broadband reflectance," J Biomed Opt 15 (6), 066019 (2010).

[21] V. Krishnaswamy, Laughney, A.M., Paulsen, K.D., Pogue, B.W., "A Dark-Field In Situ Scanning Spectroscopy Platform for Broadband Imaging of Resected Tissue," OPTICS LETTERS (2010 (in review)).

[22] D. J. Cuccia, F. Bevilacqua, A. J. Durkin, F. R. Ayers and B. J. Tromberg, "Quantitation and mapping of tissue optical properties using modulated imaging," JOURNAL OF BIOMEDICAL OPTICS 14 (2) (2009).

[23]D. J. Cuccia, F. Bevilacqua, A. J. Durkin and B. J. Tromberg, "Modulated imaging: quantitative analysis and tomography of turbid media in the spatial-frequency domain," OPTICS LETTERS 30 (11), 1354-1356 (2005).

[24] M. A. A. Neil, R. Juskaitis and T. Wilson, "Method of obtaining optical sectioning by using structured light in a conventional microscope," OPTICS LETTERS 22 (24), 1905-1907 (1997).

[25] A. Amelink, T. Christiaanse and H. J. C. M. Sterenborg, "Effect of hemoglobin extinction spectra on optical spectroscopic measurements of blood oxygen saturation," Optics Letters 34 (10), 1525-1527 (2009).

[26] R. Michels, F. Foschum and A. Kienle, "Optical properties of fat emulsions," OPTICS EXPRESS 16 (8), 5907-5925 (2008). 